\title{
Investigación interdisciplinaria e impacto social Análisis de medios sociales
}

\section{Belén Álvarez Bornstein}

Instituto de Filosofía, Consejo Superior de Investigaciones Científicas (CSIC), España | belen.alvarez@cchs.csic.es /

https://orcid.org/oooo-0003-4394-2543

\section{Michela Montesi}

Universidad Complutense de Madrid, España | mmontesi@pdi.ucm.es / https://orcid.org/oooo-0002-5509-2075

\section{Resumen}

En la actualidad se considera que la investigación interdisciplinaria (IID) tiene un mayor potencial de repercusión en la sociedad que la monodisciplinaria por abordar problemas del mundo real desde perspectivas diferentes y de mayor complejidad. Sin embargo, la evidencia de esta relación entre IID e impacto investigador es escasa. El presente trabajo tiene como objetivo comprobar si la IID tiene un comportamiento diferente a la monodisciplinaria con respecto a la atención que recibe en los medios sociales. Para ello, se analizan las menciones en varios medios (Twitter, blogs, Wikipedia, noticias e informes políticos), a través de la base de datos Altmetric.com, de la producción científica española de los años 2012-2016 indexada en la Web of Science (WoS). Los artículos fueron agrupados en 175 disciplinas, según la clasificación temática de WoS incluyendo a la categoría temática "multidisciplinary". Adicionalmente, los blogs con referencias a los artículos publicados en la categoría de Ciencias Multidisciplinares se compararon con los de las categorías de Medicina Interna y Ecología, para estudiar comparativamente la autoría y naturaleza del blog, así como la presencia de comentarios. Los resultados muestran que los artículos publicados en revistas de la categoría Ciencias Multidisciplinares están entre los más citados en los medios sociales, sobre todo en blogs y noticias. El análisis de los blogs pone de manifiesto una mayor implicación de actores ajenos a la comunidad científica con la investigación publicada en revistas de la categoría "multidisciplinary".
Palabras clave

Medios sociales Impacto social Investigación interdisciplinaria Métricas alternativas 


\section{Interdisciplinary research and societal impact: analysis of social media}

Artículo recibido: 20-09-2019. Aceptado: 04-03-2020

\section{Introducción ${ }^{1}$}

1. La investigación sobre la que informa este artículo se presentó en el Congreso IX Encuentro Ibérico EDICIC 2019, Barcelona, 9-11 de julio. Según las instrucciones del propio comité organizador del congreso, el texto completo se colgó en el repositorio E-LIS previamente a su presentación.

La investigación interdisciplinaria (en adelante IID) tiende a abordar problemas del mundo real desde perspectivas diferentes y más complejas respecto a la investigación realizada en el marco de una única disciplina, y por eso se considera con mayor potencial para aportar beneficios a la sociedad (Wagner et al., 2011; Rafols et al., 2012). Así lo entienden muchas agencias financiadoras como, por ejemplo, el National Science Foundation de Estados Unidos (Jacobs y Frickel, 2009), y algunos estudios empíricos relacionan la IID con una mayor inclinación para la innovación tecnológica (Campbell et al., 2015). Sin embargo, es escasa la evidencia de esta relación entre IID e impacto científico, tanto desde una concepción tradicional de impacto como desde una perspectiva social del mismo (Yegros-Yegros, Rafols y D'Este, 2015), y Jacobs y Frickel (2009) reclaman la realización de estudios en los cuales se analice comparativamente la IID para comprender similitudes y diferencias de esta con formas más tradicionales de producción de conocimiento.

En cuanto a los estudios realizados sobre la propia IID, desde una perspectiva sociológica, Leahey, Beckman y Stanko, (2017) identifican dos líneas principales de trabajo: la que intenta documentar los beneficios de reunir las ideas de dominios diferentes, por un lado, y la que, por otro lado, se centra en las penalizaciones para la carrera investigadora que supone emprender investigaciones de tipo interdisciplinario. Esta segunda línea sería la más proficua poniendo de manifiesto las dificultades relacionadas con la investigación que se realiza colaborativamente por diferentes disciplinas, tanto por lo que concierne a la producción como por lo que afecta a la recepción por parte de la comunidad científica. En términos de producción, habría una menor productividad 
derivada de la necesidad de coordinar equipos de procedencia dispar y del esfuerzo cognitivo implícito en la comunicación entre diferentes culturas disciplinarias. Por el lado de la recepción, cabe mencionar la confusión que puede producir este tipo de discurso científico en audiencias poco acostumbradas a los cruces disciplinarios, máxime cuando diferentes disciplinas pueden defender visiones conflictivas de los problemas estudiados (Huutoniemi y Ràfols, 2017). Los procesos de evaluación tanto cualitativa como cuantitativa también se ven afectados, y Rafols et al. (2012) ponen de manifiesto el sesgo y la discriminación que sufre este tipo de investigación a través del caso de los Innovation Studies en el Reino Unido. Este resultado se reitera en el análisis de aproximadamente 18.00o propuestas de investigación al Australian Research Council's Discovery Programme durante 5 años consecutivos, pues Bromham, Dinnage y Hua (2016) encuentran que a mayor grado de interdisciplinariedad de las propuestas corresponde una menor probabilidad de obtener financiación.

Desde la perspectiva de la bibliometría y la evaluación de la ciencia, gran parte de la investigación sobre interdisciplinariedad se ha realizado en el marco de la bibliometría tradicional, siendo el análisis de citas la herramienta de análisis más a menudo utilizada, y las ventajas y desventajas de la IID frente a la investigación monodisciplinaria o disciplinaria se han puesto de manifiesto en términos de citación e indicadores basados en la misma. Sanz-Menéndez, Bordons y Zulueta (2001) mencionan, entre las demás estrategias para el estudio de la interdisciplinariedad, la co-citación, el estudio de patrones de colaboración, o el estudio de co-ocurrencias de palabras. A pesar de las penalizaciones mencionadas anteriormente, la investigación bibliométrica ha demostrado, aunque de forma no consistente, que la investigación interdisciplinaria puede ser más exitosa en términos de citación que la monodisciplinaria. Leahey, Beckman y Stanko (2017), por ejemplo, encontraban, analizando la trayectoria y las publicaciones de unos 900 científicos estadounidenses, que la IID premiaba en términos de citación, aunque a expensas de una menor productividad. Por otro lado, Levitt y Thelwall (2008) comparan las revistas clasificadas en más de una categoría de Web of Science y Scopus (como "multidisciplinares") frente a las clasificadas en una sola categoría (mono-disciplinarias), encontrando promedios de citación parecidos en las dos clases de revistas para las Ciencias Sociales, y diferencias importantes en Biología, Ciencias de la Salud y Físicas, pues en estas áreas el promedio de citación de las revistas monodisciplinarias doblaba aproximadamente al de las multidisciplinares. En otro estudio, Larivière y Gingras (2010), quienes medían la interdisciplinariedad como el porcentaje de referencias salientes de un conjunto de artículos de Web of Science a revistas de otras disciplinas, no encontraban en general correlaciones entre el porcentaje de interdisciplinariedad y el número de citaciones. Larivière, Haustein y Börner (2015) detectaban que en una muestra de más de 9 millones de artículos interdisciplinarios publicados entre 2000 y 2012, la gran mayoría presentaba una ventaja de citación que además se extendía a los artículos que a su vez los citaran. Sin embargo, constataban importantes diferencias entre categorías tanto a favor como en contra de la IID. Chen, Arsenault y Larivière (2015) encontraban que de todos los artículos publicados en el periodo 2000-2013 en revistas de WoS, los que estaban entre el $1 \%$ más citado presentaban niveles más altos de interdisciplinariedad que los de los percentiles más bajos. Wang, Thijs y Glänzel (2015) matizan el impacto que diferentes características de la investigación pueden tener en términos de citación, diferenciando tres dimensiones de la interdisciplinariedad: la diversidad, la variedad y el equilibrio. Una mayor variedad de referencias a disciplinas diferentes y una mayor diversidad entre estas tendrían un efecto positivo en la citación a largo plazo, mientras que un mayor equilibrio, o reparto homogéneo entre referentes disciplinarios, tendría un efecto negativo a largo plazo. En general, en esta línea de investigación se ha enfatizado la repercusión que la IID tiene en términos de citación y en la comunidad académica, poniendo de manifiesto, como decíamos, diferentes matices de esta relación. 
Si en términos de impacto tradicional los resultados no terminan de ser concluyentes, los estudios realizados sobre la posible ventaja de la IID en términos de impacto socio-económico son aún más limitados, aunque no por esto menos necesarios (MolasGallart, Rafols y Tang, 2014). Los indicadores bibliométricos solo pueden mostrar una cara de la interdisciplinariedad, es decir la de la publicación en revistas científicas (Sanz-Menéndez, Bordons y Zulueta, 2001), y Wagner et al. (2011) reiteran las limitaciones de los enfoques bibliométricos para el análisis de la IID que se manifiesta, más que en las publicaciones como producto final, en un proceso dinámico a diferentes niveles y que va cambiando a lo largo del tiempo. La naturaleza cambiante de la IID se aprecia en (Chakraborty, 2018) quien, analizando la investigación en Informática en el periodo 1960-2010, detecta tres fases de evolución de una especialidad: en la fase inicial, una especialidad sería interdisciplinaria por obtener ideas de muchas otras especialidades y en la final por recibir citas de muchas especialidades diferentes. La propia dependencia de los sistemas de clasificación de los índices de citas, en particular Web of Science, sería otra gran limitación, según Wagner et al. (2011), debido a su relativa rigidez. Yegros-Yegros, Rafols y D'Este (2015) añaden que los indicadores basados en la citación no serían los más adecuados para medir el rendimiento de la IID, pues la investigación más heterodoxa suele ser poco conocida y consecuentemente poco citada, y la citación de por sí infravalora el valor de las aplicaciones prácticas de la investigación.

Si las limitaciones de los estudios basados en el análisis de citas están claramente expuestas en la literatura, las nuevas tecnologías han abierto nuevas posibilidades de medición de la interdisciplinariedad. Jiang et al. (2013), por ejemplo, miden la interdisciplinariedad a través de los grupos de discusión en el gestor de referencias social Mendeley, utilizando para tal fin la clasificación en 25 disciplinas del propio Mendeley y la información concerniente a los grupos creados por parte de los usuarios. Gomes y Dewes (2017) recurren al análisis de contenido para demostrar la interdisciplinariedad de la literatura sobre bio-combustibles publicada en WoS entre 1998 y 2007, y encuentran que, a pesar de clasificarse bibliométricamente en las áreas de Química, Ingeniería y Agricultura, esta literatura presenta abundante terminología de las Ciencias Sociales. Dong et al. (2018) proponen una batería de métodos para identificar la IID basados en el análisis de palabras clave.

El objetivo del presente trabajo es comprobar si la IID tiene un comportamiento diferente respecto a la monodisciplinaria con respecto a la atención que recibe en los medios sociales. Es de esperar que, abordando problemas del mundo real, su relevancia fuera de la comunidad académica y el interés que puede despertar en diferentes partes sociales sean mayores. Para responder a esta pregunta adoptamos una concepción operativa de interdisciplinariedad, aunque, según hemos comentado, no exenta de limitaciones, es decir las categorías contempladas en los Journal Citations Reports de WoS. Mucha literatura diferencia la interdisciplinariedad de la multidisciplinariedad y la transdisciplinariedad. Según Choi y Pak (2006, 2007), por ejemplo, la interdisciplinariedad sería diferente de la multidisciplinariedad porque, a diferencia de esta, no se limitaría a ver un mismo problema desde perspectivas diferentes sino que implicaría la definición de un marco teórico y metodológico común entre las disciplinas implicadas para la resolución de un mismo problema del mundo real. También la interdisciplinariedad sería algo diferente de la transdisciplinariedad, que pretendería solucionar problemas complejos del mundo real, a menudo en la frontera entre sistemas humanos y la naturaleza, en colaboración con las partes afectadas, incluso fuera del ámbito académico (Wickson, Carew y Russell, 2006). Desde un punto de vista bibliométrico, sin embargo, Bordons, Morillo y Gómez (2004) optan por utilizar el término interdisciplinariedad y cross-disciplinarity para referirse a los tres conceptos. En el presente estudio también optamos por un concepto operativo de interdisciplinariedad que, a efectos de comparación con otras disciplinas, identificamos con las 
revistas recopiladas en la categoría "multidisciplinary" de Web of Science (WoS). Para un conjunto de artículos publicados en revistas de WoS en el periodo 2012-16 analizamos el historial de menciones en Twitter, noticias, blogs, Wikipedia e informes de políticas a través de la herramienta Altmetric.com, atendiendo a posibles diferencias entre la categoría de multidisciplinary y las demás. La elección de estas plataformas está justificada por dos revisiones sobre el uso para fines académicos de los medios sociales (Kjellberg, Haider y Sundin, 2016; Sugimoto et al., 2017), que apuntan, por un lado, a la popularidad de Twitter en la comunidad académica, para enlazar con artículos científicos de reciente publicación y comentar en directo las presentaciones de conferencias y congresos, y a las diferentes funciones que desempeñan los blogs científicos en la comunicación científica fuera de los canales tradicionales, permitiendo, entre otras cosas, interactuar con públicos diferentes. Según los mismos estudios, la comunidad académica también utilizaría la Wikipedia, sobre todo con fines docentes, aunque sería más reacia a contribuir, debido al carácter anónimo de las contribuciones. Aun así su uso y alcance en la población son extremadamente importantes, pues según Shafee et al. (2017), quienes se centran en las páginas sobre salud publicadas en inglés (cerca de 200.000), alcanzarían los 10 millones de lecturas diarias. Las referencias a la literatura científica dentro de informes políticos serían una clara manifestación de que la investigación se ha tenido en cuenta en las tomas de decisiones (Haunschild y Bornmann, 2017). La herramienta Altmetric.com busca referencias a la literatura científica en un corpus de documentos políticos incluidos los de la Organización Mundial de la Salud o la Autoridad Europea de Seguridad Alimentaria, aunque de forma todavía imprecisa, pues en el estudio de (Tattersall y Carroll, 2018) la tercera parte de las menciones detectadas resultaron ser falsos positivos. Finalmente, los medios de comunicación de masas y las noticias de prensa siguen siendo la principal fuente de información sobre ciencia para la población en general y por esto se analizó también las referencias a la literatura científica en este marco.

\section{Metodología}

La población de estudio consiste en las publicaciones españolas recopiladas en WoS para 175 disciplinas de la edición correspondiente a las ciencias puras en el periodo 2012-2016, incluyendo la denominada como "Multidisciplinary" o Ciencias Multidisciplinares (CCMM). Los artículos pertenecientes a más de una disciplina han sido considerados una vez por cada categoría temática a la que han sido asignados por WoS en función de la revista de publicación. Para cada disciplina se obtuvo el total de publicaciones del período, el Factor de Impacto (FI) medio en el periodo analizado $\mathrm{y}$, a través de la aplicación Altmetric.com, el número de documentos mencionados al menos una vez en las siguientes plataformas: Twitter, noticias, blogs, Wikipedia e informes políticos. En el presente estudio, interpretamos que las publicaciones correspondientes a la categoría Multidisciplinary serían de carácter más interdisciplinario frente a todas las demás. Se trata de una suposición con ciertas limitaciones, pues la IID puede publicarse potencialmente en cualquier revista y, según explica Michán (2011), la auténtica interdisciplinariedad reside en la convivencia e integración de temas y fuentes de diversa procedencia que alojan los repositorios. Además, existen categorías de WoS que pueden considerarse en cierta medida interdisciplinares, como las que incluyen el término "multidisciplinary" en su denominación, por ejemplo, "Physics, multidisciplinary", o las que engloban revistas de ámbitos multidisciplinares ya consolidados, como por ejemplo "Biochemistry and Molecular Biology". Estas limitaciones deben contextualizarse en el marco exploratorio de la presente investigación y además son compartidas con mucha literatura sobre el tema. Wang y Schneider (2017) explican que existen diferentes maneras de entender la IID y operacionalizar su medición, demostrando, en su trabajo, que los diferentes indicadores adoptados resultan escasamente correlacionados los unos con los otros, y apuntan, con toda 
probabilidad, a dimensiones diferentes de la IID. De forma parecida concluyen Zhang et al. (2018) quienes, tras medir la correlación entre datos de filiación de los autores y las referencias citadas en más de 150.000 artículos publicados en PlosOne en el periodo 2007-2016, encuentran un nivel de correlación muy bajo entre las dos mediciones.

Dentro de la categoría de revistas multidisciplinares se encuentran revistas como Science y Nature que destacan especialmente por su prestigio y Factor de Impacto. Solomon, Carley y Porter (2016) investigan posibles diferencias entre las publicaciones de Nature y Science y las de tres otras revistas monodisciplinarias, Cell Biology, Physical Chemistry y Cognitive Science, aunque no encuentran diferencias significativas entre unas y otras en términos de integración de conocimiento de otras disciplinas, según se refleja en las referencias de las publicaciones analizadas, ni en difusión, en términos de citación fuera del ámbito disciplinario de las publicaciones. Estas diferencias podrían manifestarse, en su opinión, en posibles análisis altmétricos comparativos, que permitan detectar nuevas comunidades, por ejemplo, en Google Scholar, o atención por ciertos temas, sobre todo en Twitter.

Adicionalmente a los datos relativos a la repercusión en los medios sociales reseñados, se han recopilado de forma manual datos complementarios, concretamente relativos a los artículos citados en blogs, puesto que los blogs, en la literatura especializada, se consideran como herramientas poderosas de comunicación con el público, comparables con iniciativas de ciencia ciudadana (Bik y Goldstein, 2013). Enfatizando este aspecto, Mahrt y Puschmann (2014) añaden que la interacción entre autores de blogs científicos y su público se ve propiciada cuando se debaten temas de interés para muchas personas y cuando el blog se escribe de forma comprensible para el público general. En este sentido, los blogs serían un mejor indicador que Twitter de la interacción con un público no especializado, algo reiterado también en una encuesta internacional con personal de investigación sobre el uso de los medios sociales para fines académicos (Collins, Shiffman y Rock, 2016), donde Twitter resultó ser una herramienta que se utiliza principalmente para comunicarse con pares. Por otro lado, se habla de un progresivo acercamiento entre blogs y noticias de prensa científica. Según el trabajo de Bray (2019), los editoriales científicos, como respuesta a la competencia de los blogs científicos, habrían crecido en importancia en los medios de comunicación frente a las noticias científicas propiamente dichas, contribuyendo a una mayor fragmentación de la opinión pública respecto a cuestiones tan importantes como el cambio climático. El blog como espacio para dar a conocer la opinión personal sobre cuestiones de tipo científico presenta ciertos peligros, según denuncian Harvey et al. (2018) luego de analizar 45 blogs sobre cambio climático. Este equipo pone de manifiesto que a menudo la evidencia científica puede malinterpretarse o utilizarse fuera de contexto para apoyar precisamente posturas contrarias a la de la comunidad científica. Teniendo en cuenta la literatura reseñada, se recabaron datos relativos a la autoría de los blogs, que se clasificó en tres categorías (científica, no científica, no identificable), al contexto en el cual se publican (institucional, revista científica, personal, medios de comunicación, fundaciones y otros contextos), la posibilidad de comentar, y el número de comentarios. Estos datos se obtuvieron para los artículos publicados en la categoría de "Ciencias Multidisciplinares" (CCMM) y, a efectos de comparación, en las categorías de "Medicina Interna" (Med) y "Ecología" (Eco), teniendo en cuenta que tanto la salud como el medioambiente suelen despertar el interés de la ciudadanía por encima de otros temas. Para cada una de las categorías, se analizaron los tamaños muestrales indicados en la Tabla 1. 


\begin{tabular}{lccc}
\cline { 2 - 4 } & $\begin{array}{c}\text { Artículos citados } \\
\text { en blogs }\end{array}$ & $\begin{array}{c}\text { Total de citas en } \\
\text { blogs }\end{array}$ & $\begin{array}{c}\text { Muestra de } \\
\text { entradas } \\
\text { analizadas }\end{array}$ \\
\hline Ciencias Multidisciplinares & 1347 & 5430 & 360 \\
Ecología & 460 & 761 & 256 \\
Medicina Interna & 410 & 1601 & 310 \\
\hline
\end{tabular}

Tabla 1. Datos referentes a los blogs analizados

\section{Resultados}

\subsection{Atención en los medios sociales}

Los resultados muestran que Twitter es la plataforma en la que un mayor número de artículos científicos son mencionados, ya que más de un $36 \%$ de los documentos de autores afiliados a instituciones españolas publicados durante el período 2012-2016 fueron mencionados al menos una vez en Twitter. Por otro lado, los artículos citados o mencionados en el resto de las plataformas analizadas suponen menos de un $4 \%$ del total (Tabla 2) y la Wikipedia y los Informes políticos son los medios en los que la investigación tiene el menor porcentaje de aparición (alrededor de un $1 \%$ ).

\begin{tabular}{lc}
\hline Plataforma & $\begin{array}{c}\% \\
\text { Documentos }\end{array}$ \\
\hline Twitter & 36,47 \\
Noticias & 3,56 \\
Blogs & 2,90 \\
Informes Políticos & 1,10 \\
Wikipedia & 1,23 \\
\hline
\end{tabular}

Tabla 2. Porcentaje total de aparición de los artículos de investigación españoles por plataforma o medio social

De las 175 categorías analizadas en el periodo 2012-2016, por un total de 401.648 artículos, se muestran a continuación las doce disciplinas con mayor presencia en los distintos medios sociales: Twitter (Fig. 1), Blogs (Fig.2), Plataformas de noticias (Fig. 3), Informes políticos (Fig. 4) y Wikipedia (Fig. 5).

Como ya hemos comentado, Twitter es la plataforma a través de la cual se difunde mayoritariamente la investigación. Entre las disciplinas con mayor presencia en este medio se encuentran aquellas relacionadas con la biomedicina y las ciencias de la salud (Ética Médica, Atención Primaria o Alergia) y Ornitología, con más de un 80-85\% de los documentos mencionados al menos una vez. Por otro lado, la categoría de Ciencias Multidisciplinares tiene también una fuerte presencia en Twitter, puesto que alrededor del $70 \%$ de sus documentos fueron difundidos a través de esta red social. Cabe destacar que, junto con Ornitología, es la disciplina no perteneciente específicamente al ámbito de la medicina con mayor presencia en Twitter, si bien es cierto que la investigación multidisciplinar es a menudo de carácter biomédico (Figura 1).

En cuanto a las citas en blogs (Figura 2) y en noticias (Figura 3), poco menos del $15 \%$ de los artículos de la categoría de Ciencias Multidisciplinares son difundidos a 
Figura 1. Porcentaje de artículos mencionados en Twitter por disciplina

Figura 2. Porcentaje de artículos citados en blogs por disciplina
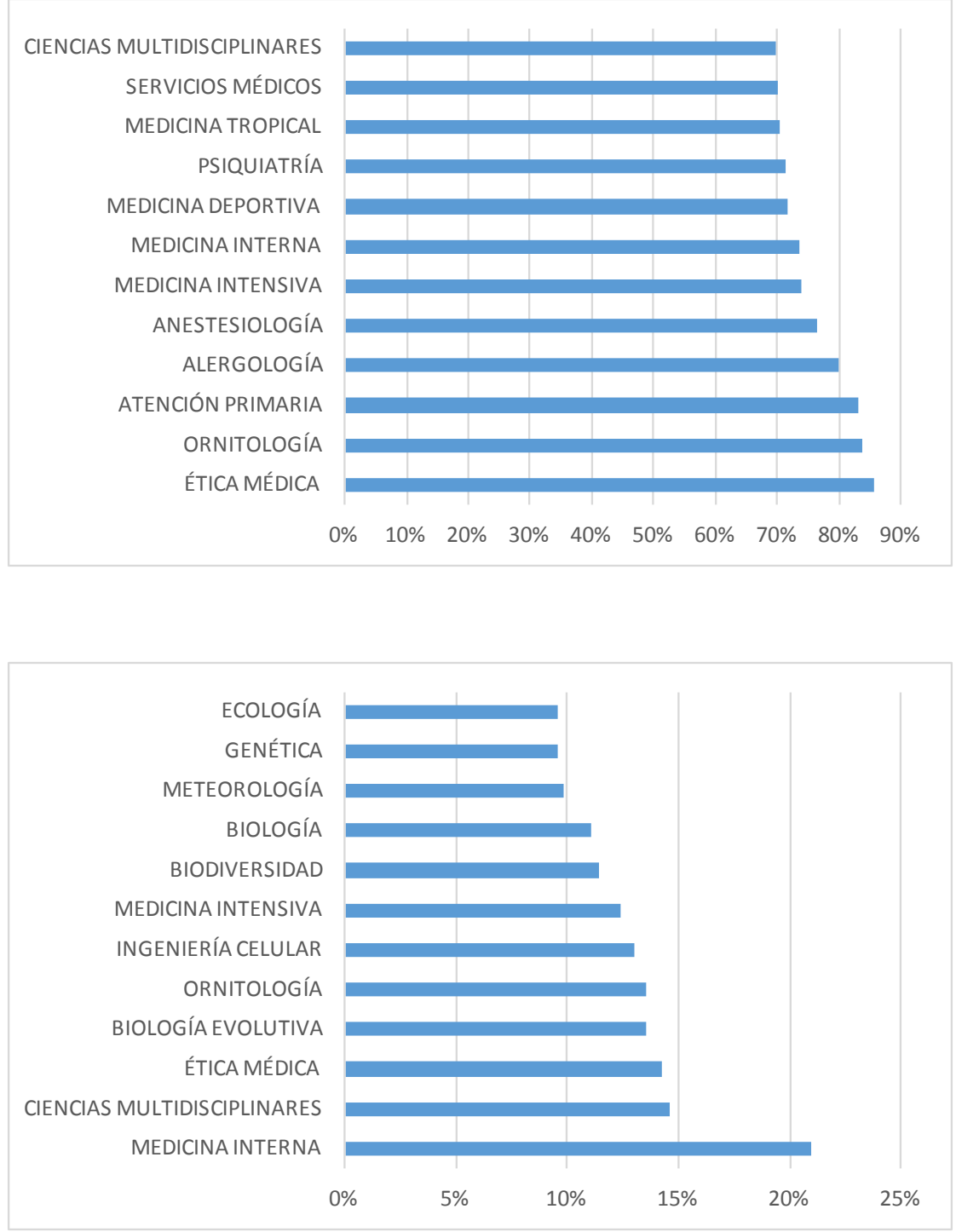

través de estos medios. Como se muestra en ambas figuras, Medicina Interna y General, con más del $20 \%$ de sus documentos mencionados tanto en blogs como en noticias, y Ciencias Multidisciplinares, con alrededor de un 15\% (blogs) y un 18\% (noticias), son las disciplinas con mayor grado de difusión. Cabe destacar que las disciplinas difundidas a través de los blogs y en las noticias son algo más variadas, puesto que entre las que tienen mayor presencia, encontramos, además de ciencias de la salud, otras relacionadas con la biología o el medio ambiente (Biología de la Evolución, Biodiversidad, Genética o Ecología).

Por último, Oncología y Nutrición y Dietética (10\%), dos temas de actualidad que suelen generar mucho interés social, se encuentran entre las disciplinas más difundidas en noticias a través de los medios de comunicación de masas (Figura 3). 

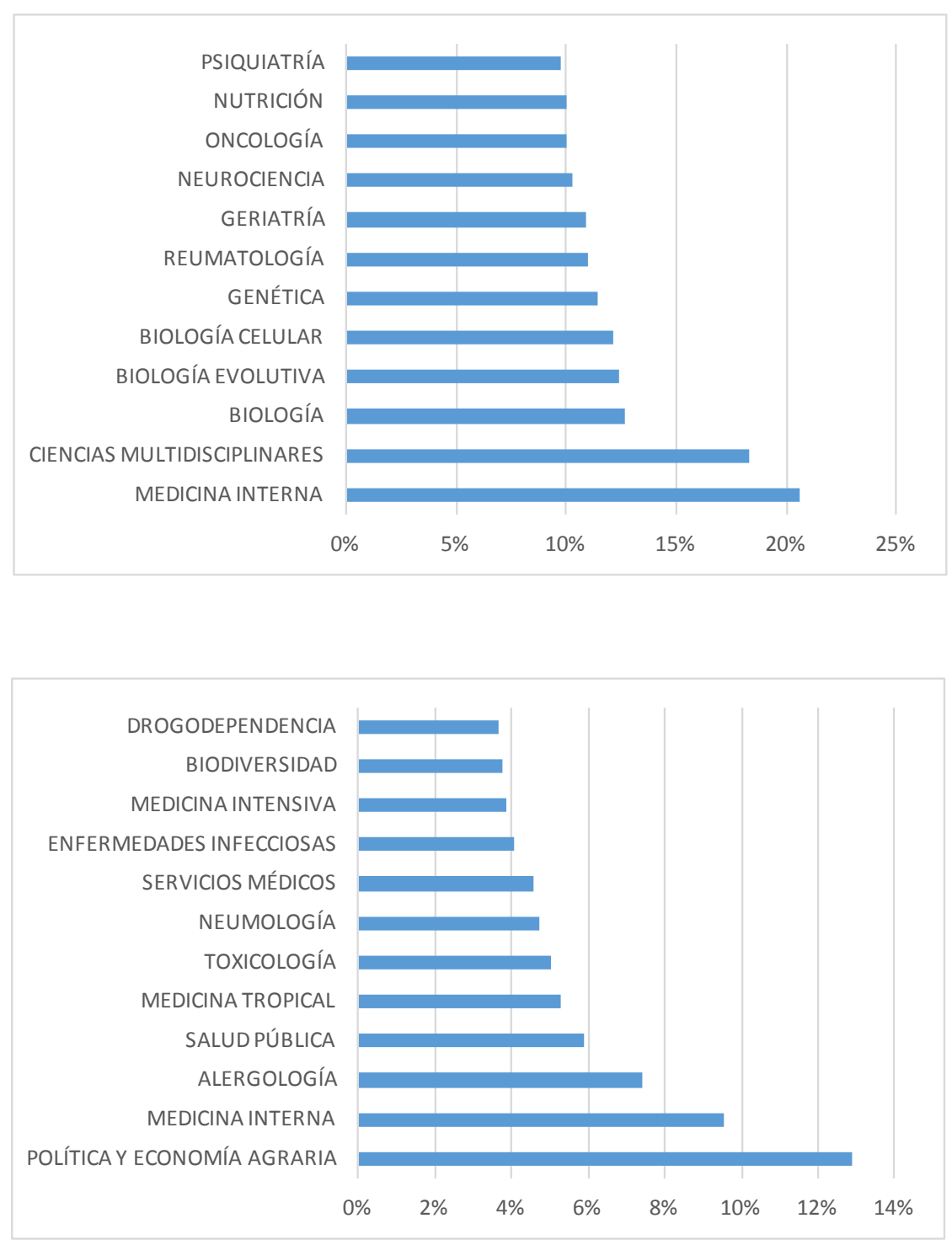

En la Figura 4 se muestra el porcentaje de artículos citados al menos una vez en informes políticos. La disciplinas con un mayor porcentaje de documentos citados en este tipo de informes son Politica y Economía Agricolas (13\%) y Medicina Interna y General (9,5\%), seguidas por otras categorías temáticas también relacionadas con la medicina y la salud pública y con el medio ambiente (Biodiversidad). No obstante, el porcentaje de artículos utilizados para la elaboración de informes políticos es muy escaso, de hecho solo el 1,1\% de los artículos fueron citados en este tipo de documentos.

En cuanto a las Ciencias Multidisciplinares, apenas un 1,5\% de sus artículos han sido citados en informes políticos, lo que puede sugerir que para elaborar este tipo de documentos se requiere de información científica más especializada.

Por último, el porcentaje de artículos citados en la Wikipedia es también escaso (Figura 5). De hecho, solo una disciplina tiene más de un $10 \%$ de sus documentos citados en esta enciclopedia colaborativa: Paleontología (15\%). Por otro lado, las disciplinas más citadas en Wikipedia pertenecen a áreas más diversas; además de categorías relacionadas con la medicina, como Medicina Interna y General o Medicina Forense, encontramos otras relacionadas con la Biología (Micología, Zoología y Ornitología) y
Figura 3. Porcentaje de artículos difundidos a través de plataformas de noticias por disciplina

Figura 4. Porcentaje de artículos citados en Informes políticos por disciplina. 
Figura 5. Porcentaje de artículos citados en la Wikipedia por disciplina

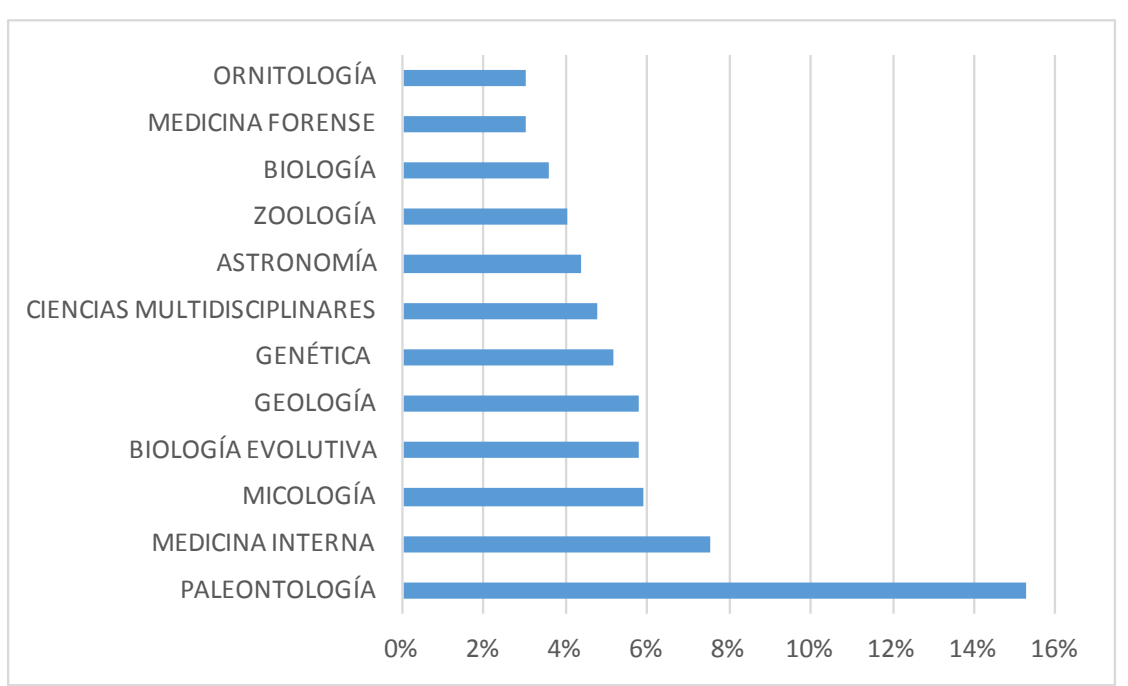

con la Geología. Como puede observarse, las Ciencias Multidisciplinares se encuentran también entre las categorías más citadas en la Wikipedia, con casi un $5 \%$ de sus artículos referenciados en ella.

\subsection{Relación entre la atención en los medios sociales y el Factor de Impacto}

Se realizaron pruebas de correlación para comprobar si existen relaciones entre la atención que recibe la literatura científica en diferentes medios sociales, y entre el Factor de Impacto medio de cada categoría y su presencia en los medios sociales. Se encontraron correlaciones significativas débiles entre FI medio y menciones en Twitter, blogs y wikis (Rho de Spearman $=0,376 ; 0,472 ; 0,415$, respectivamente), y algo más elevadas entre FI medio y atención en las noticias (Rho de Spearman=0,551). Según MacLaughlin, Wihbey y Smith (2018), los motivos para elegir qué investigaciones reflejar en la prensa tienden a prescindir del contenido del artículo y las probabilidades de aparecer en noticias serían mayores en el caso de investigaciones anunciadas en comunicados de prensa, publicadas en revistas conocidas o sobre temáticas de salud, algo que parece corroborarse en el presente análisis.

\subsection{Análisis de los blogs}

La recopilación manual de datos referentes a los blogs que citan artículos en CCMM, Med y Eco ha puesto de manifiesto una dimensión poco conocida de la difusión del conocimiento científico propiciada por las tecnologías de la información y comunicación. En cuanto a la autoría de los posts que citan artículos científicos, es necesario recalcar que en muchos casos es imposible determinar la autoría de ciertas entradas de blog porque el autor o autora quedan anónimos o se identifican con un seudónimo. La clasificación básica que realizamos pretendía medir el grado de implicación de la comunidad científica en la divulgación de la información científica en canales diferentes a los tradicionales, y el criterio que permitió discriminar entre una categoría u otra fue la existencia de algún tipo de filiación institucional. Como consecuencia, en la categoría de "autoría no científica" queda recopilado un abanico diferenciado de roles que van desde periodistas hasta profesionales de la atención primaria, profesionales de la comunicación, y simples interesados en el mundo de la ciencia, como Dienekes' Anthropology Blog. Muchas personas que escriben blogs sobre ciencia, incluso cuando lo hacen desde revistas científicas o instituciones, no tienen una 
filiación institucional propiamente dicha pero a menudo se presentan como expertos en la disciplina aportando credenciales tales como títulos de máster o doctorado. Por otro lado, los autores que se autodefinen "científicos" solo se clasificaron como tales cuando estaban afiliados con instituciones concretas y activos en investigación, según podía comprobarse a través de sus publicaciones. La Tabla 3 refleja el resultado de la clasificación de la autoría de las entradas de blog analizadas. Las CCMM destacan por el mayor porcentaje de autorías no científicas y un menor porcentaje de autorías científicas respecto a Med y Eco, poniendo de relieve una mayor implicación de personas ajenas a la comunidad científica con respecto a la investigación que se publica en este ámbito.

\begin{tabular}{lcccccc}
\cline { 2 - 7 } & \multicolumn{2}{c}{ CCMM } & \multicolumn{2}{c}{ MED } & \multicolumn{2}{c}{ ECO } \\
\cline { 2 - 7 } Tipo de autoría & $N$ & $\%$ & $N$ & $\%$ & $N$ & $\%$ \\
\hline Autoría científica & 73 & 23,1 & 109 & 39,1 & 91 & 36,4 \\
Autoría no científica & 160 & 50,6 & 120 & 43,0 & 97 & 38,8 \\
Autoría no identificable & 83 & 26,3 & 50 & 17,9 & 62 & 24,8 \\
Total parcial & 316 & & 279 & & 250 & \\
Posts no clasificables & 43 & 12,0 & 31 & 10,0 & 6 & 2,3 \\
\hline Total posts analizados & 359 & & 310 & & 256 & \\
\hline
\end{tabular}

En cuanto a la naturaleza de los blogs donde aparecen referencias a los artículos analizados, la clasificación ha simplificado una realidad muy compleja. Los medios de comunicación que se han encontrado en el análisis son a menudo portales de información o revistas exclusivamente digitales que cubren la actualidad científica, y los grandes periódicos apenas tienen una presencia en las muestras objeto de análisis. En "fundaciones" hemos recopilado todas las asociaciones que dan a conocer y fomentan la investigación en ciertas ramas de conocimiento recogiendo para ello fondos de donantes o suscriptores. En la categoría "otros" quedan incluidos blogs de organizaciones que pretenden apoyar ciertas enfermedades, como el Alzheimer, por ejemplo, hasta proyectos de investigación o blogs de empresas farmacéuticas. Muchos perfiles pueden considerarse híbridos, pues existen portales de noticias especializados en ciertas enfermedades que recuerdan a fundaciones (por ej. www.spectrumnews.org sobre autismo), mientras que las propias fundaciones se dedican a difundir noticias. Cabe añadir que entre las páginas institucionales se han clasificado algunos blogs de bibliotecas, a menudo dedicados a seleccionar publicaciones sobre temáticas concretas, o de grupos de investigación afiliados a una universidad o un centro de investigación. La Tabla 4 muestra la tipología de blogs que debaten los artículos analizados de las tres categorías, poniendo en evidencia que existen patrones específicos dependientes de las diferentes disciplinas, pues comparativamente encontramos que la literatura de CCMM se trata con mayor frecuencia en medios de comunicación respecto a las otras dos categorías. Para Med es más común que en las demás categorías aparecer en blogs correspondientes a revistas científicas, fundaciones y otras plataformas, entre las cuales están las dedicadas a difundir información contrastada en salud para ciudadanía y consumidores. Estas plataformas no se clasificaron entre los medios de comunicación porque hacen hincapié más en el concepto de "información" que de "noticias". La literatura publicada en la categoría de Eco, finalmente, se debate con mayor frecuencia que las demás categorías en blogs de revistas científicas y personales. Las CCMM, por otro lado, tienen proporcionalmente menor presencia en blogs institucionales respecto a las otras dos disciplinas, algo que no sorprende teniendo en cuenta que las instituciones se articulan en base a un modelo disciplinario más
Tabla 3. Autoría de los blogs que tratan los artículos científicos de la muestra por disciplina 
Tabla 4. Tipología de blogs donde aparecen citados los artículos de la muestra tradicional, en el que esta investigación a menudo no tiene cabida. La diferencia entre las tres categorías se ve corroborada por los resultados de la prueba del Chi-cuadrado por la que se descarta la hipótesis nula de que no exista relación entre la tipología del blog y la disciplina en la que se publica la investigación (valor del Chi-cuadrado de Pearson $=152,037 ; \mathrm{df}=10 ; \mathrm{p}$ valor $=0,000$ ), y se confirma que la investigación publicada en revistas de diferentes categorías recibe atención en diferentes medios sociales.

\begin{tabular}{lcccccc}
\cline { 2 - 7 } & \multicolumn{2}{c}{ CCMM } & \multicolumn{2}{c}{ MED } & \multicolumn{2}{c}{ ECO } \\
\cline { 2 - 7 } & $N$ & $\%$ & $N$ & $\%$ & $N$ & $\%$ \\
\hline Institucional & 35 & 10,6 & 35 & 11,9 & 41 & 16,4 \\
Revista científica & 20 & 6,0 & 54 & 18,4 & 50 & 20 \\
Personal & 84 & 25,4 & 45 & 15,4 & 89 & 35,6 \\
Medios de comunicación & 134 & 40,5 & 50 & 17,1 & 51 & 20,4 \\
Fundaciones & 18 & 5,4 & 28 & 9,6 & 11 & 4,4 \\
Otros & 39 & 11,8 & 77 & 26,3 & 6 & 2,4 \\
No identificado & 1 & 0,3 & 4 & 1,4 & 2 & 0,8 \\
\hline Total parcial & 331 & & 293 & & 250 & \\
\hline Celdas vacías & 28 & 7,8 & 17 & 5,5 & 6 & 2,3 \\
\hline Total de posts analizados & 359 & & 310 & & 256 & \\
\hline
\end{tabular}

El último aspecto analizado relativo a la presencia de la literatura científica en los blogs atañe a la capacidad de interactuar con estos contenidos y específicamente de poder comentar. Cabe explicar que no todas las entradas de blogs permiten comentar, con lo cual también se ha reflejado el total y porcentaje de blogs que sí permiten entablar un debate sobre los contenidos publicados (Tabla 5). En este sentido los blogs que discuten artículos de Eco serían los más "democráticos", pues en un $82 \%$ de los casos permiten expresar opiniones a quienes estén leyendo. Sin embargo, tan solo un $30 \%$ de los blogs que permite comentar cuenta con algún comentario en Eco, frente a un 40,7\% en Med y un 49,6 en CCMM. Finalmente, atendiendo al promedio de comentarios en las diferentes categorías, las CCMM destacan por tener el promedio más alto de comentarios $(19,1)$ y el número más alto de comentarios encontrado en la muestra para un solo post (1315).

\begin{tabular}{lcccccc}
\cline { 2 - 7 } & \multicolumn{2}{c}{ CCMM } & \multicolumn{2}{c}{ MED } & \multicolumn{2}{c}{ ECO } \\
\cline { 2 - 7 } & $N$ & $\%$ & $N$ & $\%$ & $N$ & $\%$ \\
\hline Permite comentar & 228 & 72,4 & 182 & 66,2 & 204 & 82,6 \\
Tiene comentarios & 113 & 49,6 & 74 & 40,7 & 63 & 30,9 \\
Promedio de comentarios & 19,1 & & 13,3 & & 3,1 & \\
№ máx. de comentarios & 1315 & & 465 & & 293 & \\
№ min. de comentarios & 0 & 0 & & 0 & \\
\hline
\end{tabular}

Tabla 5. Estadísticas relativas a comentarios en la muestra de blogs analizados 


\section{Discusión y conclusiones}

Este trabajo pretendía averiguar si la IID, tratando problemas de investigación más complejos y con vocación más aplicada, tiene también una mayor repercusión social. Para ello, analizamos la atención que la investigación española en Ciencias publicada en revistas de WoS entre 2012 y 2016 ha recibido en varios medios sociales. Una limitación del trabajo es que se consideró como interdisciplinaria toda la investigación publicada en la categoría de WoS titulada Ciencias Multidisciplinares, aunque estas limitaciones y otras parecidas son propias de la investigación sobre IID en general. Los resultados obtenidos apuntan a que los artículos publicados en la categoría de Ciencias Multidisciplinares suelen tener en general mucha repercusión en los medios analizados comparativamente con las demás categorías, especialmente en blogs y noticias, siendo en ambos casos la segunda categoría más citada de las 175 analizadas. Por otro lado, es menor su presencia en los informes políticos, algo que puede depender de las limitaciones de la herramienta utilizada para este fin, Altmetric.com, que solo tiene en cuenta los documentos elaborados por grandes organismos internacionales o europeos (Bornmann, Haunschild y Marx, 2016), o una menor relevancia de los resultados obtenidos en la IID para fines políticos. En cuanto a la destacable presencia en los blogs de la investigación publicada en la categoría de Ciencias Multidisciplinares, Bik y Goldstein (2013) explican que los blogs tienden a comentar artículos de revistas prestigiosas, como las incluidas entre las Ciencias Multidisciplinares (PlosOne, Nature o Science) y que, a diferencia del periodismo científico, añaden más explicaciones, contexto, análisis u opiniones expertas. En el análisis más detallado que hemos realizado de los blogs que citan las publicaciones de Ciencias Multidisciplinares, Medicina Interna y Ecología, hemos constatado que los blogs de las Ciencias Multidisciplinares destacan por su autoría, que, comparativamente, tiende a ser no científica, por su naturaleza - tienden a aparecer más a menudo en medios de comunicación-, y por generar más debate que los blogs centrados en artículos de las otras dos categorías. Es posible que la mayor presencia de las publicaciones correspondientes a la categoría de Ciencias Multidisciplinares en blogs sea un efecto derivado de las prestigiosas revistas que la conforman, algo confirmado por la existencia de una correlación general moderada entre el FI medio de las categorías de WoS y la presencia en estos medios sociales. Sin embargo, cuando analizamos la actividad generada alrededor de estos contenidos y materializada, concretamente, en los comentarios, existen diferencias importantes a favor de las Ciencias Multidisciplinares que no pueden achacarse a la influencia de las revistas y sus indicadores de impacto y que parecen depender más bien de la propia naturaleza de la investigación. En este sentido las publicaciones de la categoría Ciencias Multidisciplinares suelen despertar más interés y generar más debate. Todos estos aspectos corroboran una posible mayor repercusión fuera de la comunidad científica, aunque sea difícil hablar de impacto social, pues este concepto está pendiente aún de una definición más concreta (Bornmann, 2013). 


\section{Q Referencias Bibliográficas}

» Bik, Holly M. y Miriam C. Goldstein. 2013. An introduction to social media for scientists. En PLoSbiology. Vol. 11, no. 4, e1001535. <https://doi.org/10.1371/journal.pbio.1001535>

»Bordons, María, Fernanda Morillo e Isabel Gómez. 2004. Analysis of cross-disciplinary research through bibliometric tools. En Moed HenkF., Wolfgang Glänzel y Ulrich Schmoch, eds. Handbook of quantitative science and technology research. Dordrecht: Springer. p. 437-456. <https://doi.org/10.1007/1-4020-2755-9_20>.

"Bornmann, Lutz. 2013. What is societal impact of research and how can it be assessed? A literature survey. En Journal of the American Society for Information Science and Technology. Vol. 64, no. 2, 217-233. <https://doi.org/10.1002/asi.22803>

» Bornmann, Lutz, Robin Haunschild y Werner Marx. 2016. Policy documents as sources for measuring societal impact: how often is climate change research mentioned in policy-related documents? En Scientometrics. Vol. 109, no. 3, 14771495. <https://doi.org/10.1007/s11192-016-2115-y>

»Bray, Nancy. 2019. How Do Online News Genres Take Up Knowledge Claims From a Scientific Research Article on Climate Change? En Written Communication. Vol. 36, no.1, 155-189. <https://doi.org/10.1177/0741088318804822>

» Bromham, Lindell, Russel Dinnage y Xia Hua. 2016. Interdisciplinary research has consistently lower funding success. En Nature. Vol. 534, 684-687.

"Campbell, David, Philippe Deschamps, Grégoire Côté, Guillaume Roberge, Christian Lefebvre y Éric Archambault. 2015. Application of an "interdisciplinarity" metric at the paper level and its use in a comparative analysis of the most publishing ERA and non-ERA universities. En International Conference on Science and Technology Indicators. (20th: 2 - 4 Septiembre 2015: Lugano, Switzerland) Trabajos presentados. Montreal: Science-Metrix. <https://www.science-metrix. $\mathrm{com} /$ ?q=en/publications/conference-presentations/application-of-an-interdisciplinarity-metric-at-the-paper>[Consulta: 10 septiembre 2019].

"Chakraborty, Tanmoy. 2018. Role of interdisciplinarity in computer sciences: quantification, impact and life trajectory. En Scientometrics. Vol. 114, no. 3, 10111029. <https://doi.org/10.1007/s11192-017-2628-z>

"Chen, Shiji, Clément Arsenault y Vincent Larivière. 2015. Are top-cited papers more interdisciplinary? En Journal of Informetrics. Vol. 9, no. 4, 1034-1046. $<$ https://doi.org/10.1016/j.joi.2015.09.003>

»Choi, Bernard C.K. y Anita W.P. Pak. 2006. Multidisciplinarity, interdisciplinarity and transdisciplinarity in health research, services, education and policy: 1. Definitions, objectives, and evidence of effectiveness. En Clinical \& Investigative Medicine. Vol. 29, no. 6, 351-364.

»Choi, Bernard C.K. y Anita W.P. Pak. 2007. Multidisciplinarity, interdisciplinarity, and transdisciplinarity in health research, services, education and policy: 2 . Promotors, barriers, and strategies of enhancement. En Clinical \& Investigative Medicine. Vol. 30, no. 6, e224-e232. <https://doi.org/10.25011/cim.v3oi6.2950>

"Collins, Kimberley, David Shiffman y Jenny Rock. 2016. How are scientists using social media in the workplace? En PlosOne. Vol. 11, no. 10, e016268o. <https:// dx.doi.org/10.1371\%2Fjournal.pone.0162680> 
»Dong, Kun, Haiyun Xu, Rui Luo, Ling Wei y Shu Fang. 2018. An integrated method for interdisciplinary topic identification and prediction: a case study on information science and library science. En Scientometrics. Vol. 115, no. 2, 849-868. <https://doi.org/10.1007/s11192-018-2694-x>

"Gomes, Janaína y Homero Dewes. 2017. Disciplinary dimensions and social relevance in the scientific communications on biofuels. En Scientometrics. Vol. 110, no. 3, 1173-1189. <https://doi.org/10.1007/s11192-016-2233-6>

» Harvey, Jeffrey A., Daphne van den Berg, Jacintha Ellers, Remko Kampen, Thomas W. Crowther, Peter Roessingh, Bart Verheggen, Rascha J. M. Nuijten, Eric Post, Stephan Lewandowsky, lan Stirling, Meena Balgopal, Steven C. Amstrup y Michael E. Mann. 2018. Internet blogs, polar bears, and climate-change denial by proxy. En BioScience. Vol. 68, no. 4, 281-287. <https://doi.org/10.1093/biosci/ bix133>

»Haunschild, Robin y Lutz Bornmann. 2017. How many scientific papers are mentioned in policy-related documents? An empirical investigation using Web of Science and Altmetric data. En Scientometrics. Vol. 110, no. 3, 1209-1216. <https://doi.org/10.1007/s11192-016-2237-2>

» Huutoniemi, Katri Ilona e Ismael Ràfols. 2017. Interdisciplinarity in research evaluation. En Frodeman, Robert, Julie Thompson Klein y Roberto Carlos Dos Santos Pacheco, eds. The Oxford Handbook of interdisciplinarity. 2nd ed. Oxford: Oxford University Press. p. 498-512. <https://helda.helsinki.fi//bitstream/ handle/10138/309928/SSRN_id2818321.pdf?sequence=1> [Consulta: 27 marzo 2020]

» Jacobs, Jerry A. y Scott Frickel. 2009. Interdisciplinarity: A critical assessment. En Annual Review of Sociology. Vol. 35, 43-65. <https://doi.org/10.1146/annurevSOC-070308-115954>

" Jiang, Jiepu, Chaoqun Ni, Daqing He y Wei Jeng. 2013. Mendeley group as a new source of interdisciplinarity study: how do disciplines interact on Mendeley? En JCDL '13: Proceedings of the 13th ACM/IEEE-CS joint conference on Digital libraries. (13th: 22-26 Julio 2013: Indianapolis, Indiana). Trabajos presentados. Nueva York: Association for Computing Machinery. p. 135-138. <https://doi. org/10.1145/2467696.2467738>

" Kjellberg, Sara, Jutta Haider y Olof Sundin. 2016. Researchers' use of social network sites: A scoping review. En Library \& Information Science Research. Vol. 38, no. 3, 224-234. <https://doi.org/10.1016/j.lisr.2016.08.008>

» Larivière, Vincent e Yves Gingras. 2010. On the relationship between interdisciplinarity and scientific impact. En Journal of the American Society for Information Science and Technology. Vol. 61, no. 1, 126-131. <https://doi.org/10.1002/ asi.21226>

"Larivière, Vincent, Stefanie Haustein y Katy Börner. 2015. Long-distance interdisciplinarity leads to higher scientific impact. En PlosOne. Vol. 10 no. 3 , e0122565. <https://doi.org/10.1371/journal.pone.0122565>

"Leahey, Erin, Christine M. Beckman y Taryn L. Stanko. 2017. Prominent but less productive: The impact of interdisciplinarity on scientists' research. En Administrative Science Quarterly. Vol. 62, no. 1, 105-139. <https://doi. org/10.1177/0001839216665364>

»Levitt, Jonathan M. y Mike Thelwall. 2008. Is multidisciplinary research more highly cited? A macrolevel study. En Journal of the American Society for Information Science and Technology. Vol. 59, no. 12, 1973-1984. <https://doi.org/10.1002/asi.20914> 
"MacLaughlin, Ansel, John Wihbey y David A. Smith. 2018. Predicting News Coverage of Scientific Articles. En Proceedings of the Twelfth International AAAI Conference on Web and Social Media. Vol. 13, no. 01, 158-169. <https://aaai.org/ocs/index. php/ICWSM/ICWSM18/paper/view/17805/17010> [Consulta: 27 marzo 2020]

" Mahrt, Merja y Cornelius Puschmann. 2014. Science blogging: An exploratory study of motives, styles, and audience reactions. En Journal of Science Communication. Vol. 13, no. 3, A05. <https://doi.org/10.22323/2.13030205>

» Michán, Layla. 2011. Cienciometría, información e informática en ciencias biológicas: enfoque interdisciplinario para estudiar interdisciplinas. En Ludus Vitalis. Vol. 19, no. 35, 239-243. <http://www.ludus-vitalis.org/ojs/index.php/ludus/ article/view/214/210> [Consulta: 27 marzo 2020]

» Molas-Gallart, Jordi, Ismael Rafols y Puay Tang. 2014. On the Relationship between Interdisciplinarity and Impact: Different modalities of interdisciplinarity lead to different types of impact. En The Journal of Science Policy and Research Management. Vol. 29, no 2-3, 69-89. <https://doi.org/10.20801/jsrpim.29.2_3_69>

» Rafols, Ismael, Loet Leydesdorff, Alice O’Hare, Paul Nightingale y Andy Stirling. 2012. How journal rankings can suppress interdisciplinary research: A comparison between Innovation Studies and Business \& Management. En Research Policy. Vol. 41, no. 7, 1262-1282. <https://doi.org/10.1016/j.respol.2012.03.015>

"Sanz-Menéndez, Luis, María Bordons y M. Ángeles Zulueta. 2001. Interdisciplinarity as a multidimensional concept: its measure in three different research areas. En Research Evaluation. Vol. 10, no. 1, 47-58. <https://doi. org/10.3152/147154401781777123>

"Shafee, Thomas, Gwinyai Masukume, Lisa Kipersztok, Diptanshu Das, Mikael Häggström y James Heilman. 2017. Evolution of Wikipedia's medical content: past, present and future. En Journal of Epidemiology and Community Health. Vol. 71, no. 11, 1122-1129. <http://dx.doi.org/10.1136/jech-2016-208601>

» Solomon, Gregg E.A., Stephen Carley y Alan L. Porter. 2016. How multidisciplinary are the multidisciplinary journals Science and Nature? En PlosOne. Vol. 11, no. 4, e0152637. <https://doi.org/10.1371/journal.pone.0152637>

"Sugimoto, Cassidy R., Sam Work, Vincent Larivière y Stefanie Haustein. 2017. Scholarly use of social media and altmetrics: A review of the literature. En Journal of the Association for Information Science and Technology. Vol. 68, no. 9, 20372062. <https://doi.org/10.1002/asi.23833>

» Tattersall, Andy y Christopher Carroll. 2018. What can altmetric.com Tell Us about Policy citations of research? An analysis of altmetric.com Data for research articles from the University of Sheffield. En Frontiers in Research Metrics and Analytics. Vol. 2, 9. <https://doi.org/10.3389/frma.2017.00009>

»Wagner, Caroline S., J. David Roessner, Kamau Bobb, Julie Thompson Klein, Kevin W. Boyack, Joann Keyton, Ismael Rafols y Katy Börner. 2011. Approaches to understanding and measuring interdisciplinary scientific research (IDR): $A$ review of the literature. En Journal of informetrics. Vol. 5 no. 1, 14-26. <https://doi. org/10.1016/j.joi.2010.06.004>

»Wang, Jian, Bart Thijs y Wolfgang Glänzel. 2015. Interdisciplinarity and impact: Distinct effects of variety, balance, and disparity. En PlosOne. Vol. 10, no. 5, e0127298. <https://doi.org/10.1371/journal.pone.0127298>

»Wang, Qi y Jesper W. Schneider. 2017. Consistency of interdisciplinarity indicators. En Issi 2017 - 16th International Conference on Scientometrics and Informetrics, 
Conference Proceedings. (16 $6^{\text {th }}: 16-20$ octubre 2017: Wuhan, China). Aarhus: International Conference on Scientometrics and Informetrics. p. 1406-1417.

"Wickson, Fern, Anna L Carew y A. Wendy Russell. 2006. Transdisciplinary research: characteristics, quandaries and quality. En Futures. Vol. 38, no. 9, 10461059. <https://doi.org/10.1016/j.futures.2006.02.011>

»Yegros-Yegros, Alfredo, Ismael Rafols y Pablo D’Este. 2015. Does interdisciplinary research lead to higher citation impact? The different effect of proximal and distal interdisciplinarity. En PlosOne. Vol. 10, no. 8, e0135095. <https://doi. org/10.1371/journal.pone.0135095>

"Zhang, Lin, Beibei Sun, Zaida Chinchilla-Rodríguez, Lixin Chen y Ying Huang. 2018. Interdisciplinarity and collaboration: on the relationship between disciplinary diversity in departmental affiliations and reference lists. En Scientometrics. Vol. 117, no. 1, 271-291. <https://doi.org/10.1007/s11192-018-2853-0> 
